\title{
CORRELATION OF ION CONTENTS WITH ETHYLENE PRODUCTION DURING THE RIPENING PROCESS OF SUGARCANE (SACCHARUM OFFICINARUM L.) WITH THE APPLICATION OF CHEMICAL RIPENERS
}

\author{
TUFAIL, M. - HuSSAIN, K.* \\ Department of Botany, University of Gujrat (UOG), Gujrat, Pakistan \\ *Corresponding author \\ e-mail: khalid.hussain@uog.edu.pk \\ (Received 27 $7^{\text {th }}$ Apr 2020; accepted $11^{\text {th }}$ Aug 2020)
}

\begin{abstract}
Chemical ripeners (Ethephon, Glyphosate and Sulfometuron-methyl) were used as foliar sprays on four sugarcane cultivars (HSF-242, NSG-311, HSF-240, NSG-555) to find their effects on quality, potassium $\left(\mathrm{K}^{+}\right)$and chloride $\left(\mathrm{Cl}^{-}\right)$contents and ethylene production. Chemical ripeners significantly increased the sucrose contents, juice purity, recovery \% cane and sugar yield based on the mean data of two years. All the sugarcane cultivars sprayed with chemical ripeners responded well regarding early cane maturity and high production of sugar. Variations in antioxidant activities i.e. peroxidase (POD) and catalases (CAT) were also noted indicating the stimulating effects of various chemical ripeners. There was a negative correlation of $\mathrm{K}^{+}$and $\mathrm{Cl}^{-}$with ethylene production $\mathrm{r}=-0.8915,-0.7773$, respectively. $\mathrm{K}^{+}$had a positive correlation with $\mathrm{Cl}^{-}$contents $(\mathrm{r}=0.92205)$. Sugar yielding attributes had positive correlations with ethylene while, $\mathrm{K}^{+}$and $\mathrm{Cl}^{-}$had negative correlations. Upon reaching maturity, ethylene production increased while, $\mathrm{K}^{+}$and $\mathrm{Cl}^{-}$contents in cane juice decreased resulting in high sucrose contents in sugarcane. It was concluded that chemical ripeners were useful reaching early cane maturity in sugarcane cultivars which were considered mid or late maturing. Chemical ripeners also increased the sucrose contents with high sugar yield by affecting various variables linked to cane maturity. These outcomes can be utilized by researchers and plant breeders to develop early cane maturity varieties during sugarcane variety development programs.

Keywords: potassium, chloride, antioxidant activities, sucrose, sugar yield
\end{abstract}

\section{Introduction}

Sugarcane (Saccharum officinarum L.) is considered as an industrial crop for the production of sugar (Neliana et al., 2019). Sugarcane is cultivated on 1132 thousand hectares with a total production of 65,475 thousand tons (Manzoor et al., 2019). Sugarcane is rich in sucrose which is accumulated in stalk internodes and is used to manufacture many industrial goods such as furfural, alcohol, dextrans and some other natural pharmaceutical products (Ma et al., 2005).

Chemical ripeners (Ethephon, ethyl-trinexapac, glyphosate and sulfometurom methyl) are classified as growth retardants and growth inhibitors (Leite et al., 2010). Chemical ripening of sugarcane is profitable due to high sugar production throughout the world (Dalley and Richard, 2010). The key benefit of chemical ripeners is that they can suppress leaf and stalk growth more rapidly than natural mechanism (Van Heerden et al., 2015). Applications of Ethephon has accelerated the ripening process of sugarcane, its yield and it inhibited the flowering (Chong et al., 2010; De Almeida and Caputo, 2012).

The chemical ripener glyphosate is an amino acid synthesis inhibitor and it has been extensively used to increase sucrose contents in sugarcane (Solomon and Li, 2004). Glyphosate is a widely used herbicide throughout the world because it is an efficient killer of weeds, less toxic and available at low cost (Goscinny and Hanot, 2012). Leite et al. (2009) showed that the applications of ripeners used for early harvest sugarcane 
resulted in an increase of quality, sugar yield which positively increased the profit per unit area. El-Hamd et al. (2013) noted that glyphosate applications helped to increase the total soluble solids in juice and other quality parameters and sucrose contents. Sulfometuron-methyl is a grass herbicide that was promising as a chemical ripener at low rates of application (Almodares et al., 2013). Many studies reported that sulfometuron-methyl has good potential of ripening in sugarcane varieties and it causes no damage in sugarcane production (Silva et al., 2007; Leite et al., 2010).

Chemical ripeners or herbicides can affect the production of ethylene that can induce early maturity. Lee and Dumas (1982) found the changes in ethylene production in Tobacco with the applications of Glyphosate. Glyphosate beneficially increased the sucrose contents in sugarcane (McDonald et al., 2001). Changes in ethylene level and its perception, directly or indirectly regulate the lifespan of plants. Ethylene is tightly correlated with the biosynthesis of volatile organic compounds (Iqbal et al., 2017). There are various nutrients that have a role in sugarcane maturity. Watanabe et al. (2016) showed that potassium $\left(\mathrm{K}^{+}\right)$ and chloride $\left(\mathrm{Cl}^{-}\right)$contents show a relationship with sucrose contents. They have negative correlation with sucrose contents in sugarcane. The ripening of fruits is a unique coordination of various biochemical and developmental pathways regulated by ethylene, which affects color, texture, nutritional quality and aroma of fruits (Barry and Giovannoni, 2007).

Ethylene production and concentration of nutrients mainly potassium and chloride affect the sugar contents during maturity but so far, their relationship has not been established for selected sugarcane cultivars in Pakistan. This study was conducted to find the effect of chemical ripeners on sugar production and relationship between ethylene production with cation and anion contents in sugarcane.

\section{Materials and methods}

Experiments were carried out at Shakarganj Sugar Research Institute (SSRI) Jhang and University of Gujrat, Pakistan during 2018-19. Planting of four sugarcane cultivars i.e. HSF-242, NSG-311, HSF-240 and NSG-555 was done in autumn 2018 and harvesting was carried out in autumn 2019. These cultivars were extensively cultivated in the country due to better growth, yield performance and good ratooning abilities with resistance against major diseases of sugarcane. These cultivars have not yet been evaluated to find the effect of chemical ripeners. Experimental design was RCBD (Plot size $40 \times 40$ feet beds) with four replicates. Seed rate was 75000 double-bedded setts per hectares with 2.5 feet row spacing.

Each chemical ripener was used at cane formation and elongation phase that has not been studied so far in Pakistan and for selected cultivars. The following treatments were carried out calculated on hectare basis:

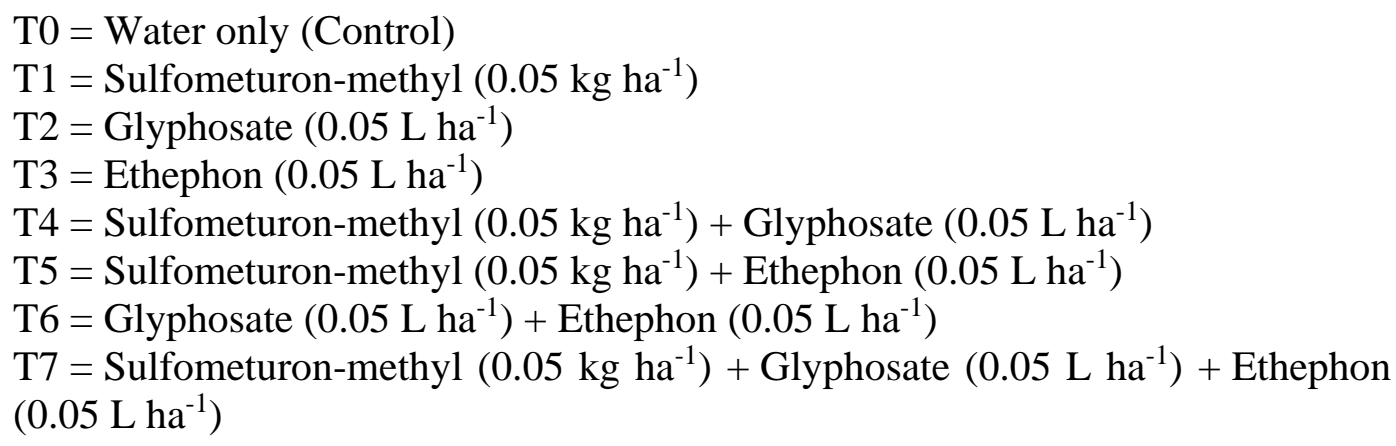




$$
-65 \text { - }
$$

Four cane samples were collected for the study of following the quality parameters, sugar yielding and antioxidant activities in autumn 2019:

1. Sucrose percentage $(\%)$

2. Juice purity $(\%)$

3. Sugar recovery $\%$ cane

4. Sugar yield $\left(\mathrm{t} \mathrm{ha}^{-1}\right)$

5. Antioxidant activities (POD and CAT)

6. Determination of $\mathrm{K}^{+}$and $\mathrm{Cl}^{-}$contents

7. Determination of ethylene production in leaf sheaths

Brix reading was measured using Brix hydrometer as a percentage of the total soluble solids in juice $(\mathrm{w} / \mathrm{w})$ by hand refractometer. Sucrose percentage was calculated from $100 \mathrm{~cm}^{3}$ of juice using the following equation described by Horwitz (1976):

$$
\text { Sucrose percentage }=\text { direct reading of Saccharimeter } \times 1.04
$$

Where 1.04 is a factor depending on the length of Saccharimeter's tube.

Juice Purity was calculated using the following equation:

$$
\text { Purity percentage }=(\text { sucrose percentage } / \text { Brix reading }) \times 100
$$

Theoretical sugar recovery was calculated according to the formula described by Legendre and Henderson (1972):

$$
\text { Sugar recovery percentage }=[S-0.4(B-S)] \times 0.73
$$

Where: $\mathrm{B}=$ Brix percentage, $\mathrm{S}=$ sucrose percentage, 0.4 and 0.73 constant factors.

$$
5 \text { - Sugar yield }(\text { ton } / \text { fed })=\text { net cane yield } \times \text { theoretical sugar recovery }
$$

Total sugar yield was calculated for each treatment by using the following method:

$$
\text { Total sugar }\left(\mathrm{t} \mathrm{ha}^{-1}\right)=\text { Sugar recovery } \times \text { Stripped-cane yield } / 100
$$

Antioxidant activities i.e. Peroxidase (POD) and catalases (CAT) were determined by the procedure of Chance and Maehly (1955). Potassium $\left(\mathrm{K}^{+}\right)$contents in cane juice were determined with a flame photometer (model PFP7, Jenway Staffordshire, UK). Chloride $\left(\mathrm{Cl}^{-}\right)$contents in the juice of sugarcane were determined by a chloride meter. Leaf samples were collected and were immediately put into ethylene bottles and sealed.

Sugarcane leaves were kept in container for $5 \mathrm{~h}$ to measure ethylene production by portable Ethylene meter. These measurements were repeated five times and mean values were calculated for $2^{\text {nd }}$ to $5^{\text {th }}$ leaf.

Data were subjected to analysis of variance for three factor factorial in Minitab (Version: 19.2.0, Coventry, UK) and significant mean separation was done at $\mathrm{P} \leq 0.05$ using Tukey's test. 


\section{Results}

\section{Sucrose contents $(\%)$}

Effect of chemical ripeners was highly significant in sugarcane for sucrose contents (Table 1). The interaction of ripeners $\mathrm{x}$ cultivars was also significant. Higher sucrose contents $(12.6 \%)$ were noted in cultivar NSG-555 with $\mathrm{T}_{2}$ (Glyphosate $\left(0.05 \mathrm{~L} \mathrm{ha}^{-1}\right)$. Overall, Glyphosate and Sulfometuron-methyl $\left(0.05 \mathrm{~kg} \mathrm{ha}^{-1}\right)+$ Ethephon $\left(0.05 \mathrm{~L} \mathrm{ha}^{-1}\right)$ applications was best for the enhancement of sucrose contents (Fig. 1A). Chemical ripeners applied as separate treatments were more effective than combined applications (Table 2).

Table 1. Mean squares (MS) from the analysis of variance (ANOVA) of sugarcane cultivars for various attributes under the applications of chemical ripeners

\begin{tabular}{|c|c|c|c|c|c|c|c|c|c|}
\hline $\begin{array}{c}\text { Source } \\
\text { Main effects }\end{array}$ & df & $\begin{array}{l}\text { Sucrose } \\
\text { contents }\end{array}$ & $\begin{array}{c}\text { Juice } \\
\text { purity }(\%)\end{array}$ & $\begin{array}{l}\text { Sugar } \\
\text { recovery } \\
\% \text { cane }\end{array}$ & $\begin{array}{c}\text { Total sugar } \\
\text { yield }\end{array}$ & $\begin{array}{c}\text { Peroxidase } \\
\text { (POD) } \\
\text { activities }\end{array}$ & $\begin{array}{c}\text { Catalases } \\
\text { (CAT) } \\
\text { activities }\end{array}$ & $\begin{array}{l}\mathbf{K}^{+} \text {in } \\
\text { Juice }\end{array}$ & $\mathrm{Cl}^{-}$in juice \\
\hline Replicates & 3 & $24.345^{*}$ & $11.56 * *$ & $0.342^{*}$ & $67.453 * *$ & $0.003 *$ & $0.045^{*}$ & $0.023 *$ & $0.011^{*}$ \\
\hline $\begin{array}{l}\text { Ripeners } \\
\text { (Rip) }\end{array}$ & 7 & $62.579 * *$ & $52.524 * * *$ & $0.522 * *$ & $479.487 * * *$ & $0.005^{* *}$ & $0.076 * *$ & $0.067 * *$ & $0.043 * *$ \\
\hline $\begin{array}{c}\text { Cultivars } \\
\text { (cv) }\end{array}$ & 3 & $1379.752 * *$ & $626.596 * *$ & $6.256^{* *}$ & $8236.089 * *$ & $0.076^{* *}$ & $0.150 * *$ & $0.146^{* *}$ & $0.231 * *$ \\
\hline $\begin{array}{c}\text { Interactions } \\
\text { cv x Rip }\end{array}$ & 21 & $17.396 * *$ & $12.888 * * *$ & $0.121 * *$ & $34.211 * *$ & $8.823 * *$ & $0.005^{*}$ & $0.098^{*}$ & $0.067 *$ \\
\hline Error & 93 & 7.943 & 2.614 & 0.043 & 13.011 & 0.001 & 0.002 & 0.076 & 0.058 \\
\hline Total & 127 & & & & & & & & \\
\hline
\end{tabular}

\section{Juice purity (\%)}

It was noted from the results that the effect of chemical ripeners was highly significant for juice purity $\%$ of sugarcane. Variations among cultivars as well as the interaction between ripeners $\mathrm{x}$ cultivar were also highly significant (Table 1). Higher juice purity $\%$ was calculated in NSG-555 and minimum juice purity was present in HSF-242 (Table 2). Sulfometuron-methyl $\left(0.05 \mathrm{~kg} \mathrm{ha}^{-1}\right)+$ Ethephon $\left(0.05 \mathrm{~L} \mathrm{ha}^{-1}\right)$ application increased juice purity up to $83.8 \%$ in HSF-240 (Fig. 1B).

\section{Sugar recovery \% from cane}

Sugar recovery \% from cane significantly increased with the applications of chemical ripeners (Table 1). Ethephon $\left(0.05 \mathrm{~L} \mathrm{ha}^{-1}\right)$ treatments were more effective as compared to other applications (Fig. 1C). Higher sugar recovery \% from cane (12.12) was calculated in NSG-555 following Ethephon $\left(0.05 \mathrm{~L} \mathrm{ha}^{-1}\right)$ treatment. All the chemical ripener treated plants showed higher sugar recovery \% from cane as compared to control (Fig. 1C).

\section{Sugar yield ( ha $\left.^{-1}\right)$}

Sugar yield was highly significantly affected by the applications of chemical ripeners in all the cultivars of sugarcane. Effect of chemical ripeners for cultivars and the effect of their interaction ripeners $\mathrm{x}$ cultivar were also highly significant (Table 1). Ethephon $\left(0.05 \mathrm{~L} \mathrm{ha}^{-1}\right)$ treatments were the best for the enhancement of sugar yield (Fig. 1D). Higher sugar yield (116.85 $\left.\mathrm{t} \mathrm{ha}^{-1}\right)$ was obtained from NSG-555 with Ethephon (0.05 L ha ${ }^{1}$ ) treatments (Table 2). 
Table 2. Means comparison for different variables of sugarcane using Tukey's test

\begin{tabular}{|c|c|c|c|c|c|c|c|c|c|}
\hline Cultivar & Treatments & $\begin{array}{c}\begin{array}{c}\text { Sucrose } \\
\text { contents } \\
(\%)\end{array} \\
\end{array}$ & $\begin{array}{c}\text { Juice } \\
\text { purity } \\
(\%)\end{array}$ & $\begin{array}{l}\text { Sugar } \\
\text { recovery } \\
\% \text { cane }\end{array}$ & $\begin{array}{c}\text { Sugar } \\
\text { yield } \\
\left(\mathbf{t ~ h a}^{-1}\right)\end{array}$ & $\begin{array}{l}\text { Peroxidase } \\
\text { (POD) } \\
\text { activities }\end{array}$ & $\begin{array}{c}\text { Catalases } \\
\text { (CAT) } \\
\text { activities }\end{array}$ & $\mid \begin{array}{c}\mathrm{K}^{+} \text {contents } \\
\text { in juice }\end{array}$ & $\begin{array}{l}\mathrm{Cl}^{-} \text {contents } \\
\text { in juice }\end{array}$ \\
\hline \multirow{9}{*}{ HSF-242 } & T0 & $9.7 \mathrm{~B}$ & $71.82 \mathrm{~B}$ & $9.12 \mathrm{E}$ & $74.32 \mathrm{BC}$ & $0.892 \mathrm{D}$ & $1.027 \mathrm{~B}$ & $54.51 \mathrm{~A}$ & $34.21 \mathrm{~A}$ \\
\hline & $\mathrm{T} 1$ & $10.9 \mathrm{~A}$ & $71.57 \mathrm{~B}$ & $9.97 \mathrm{D}$ & $72.91 \mathrm{C}$ & $0.895 \mathrm{D}$ & $1.037 \mathrm{~A}$ & $40.67 \mathrm{~B}$ & $26.23 \mathrm{~B}$ \\
\hline & $\mathrm{T} 2$ & $10.2 \mathrm{~A}$ & $74.05 \mathrm{~A}$ & $10.19 \mathrm{C}$ & $76.81 \mathrm{~B}$ & $0.912 \mathrm{C}$ & $1.025 \mathrm{~B}$ & $32.45 \mathrm{C}$ & $18.09 \mathrm{C}$ \\
\hline & $\mathrm{T} 3$ & $10.3 \mathrm{~A}$ & $76.05 \mathrm{~A}$ & $11.23 \mathrm{~A}$ & $86.05 \mathrm{~A}$ & $0.991 \mathrm{BC}$ & $1.042 \mathrm{~A}$ & $37.56 \mathrm{C}$ & $19.02 \mathrm{C}$ \\
\hline & $\mathrm{T} 4$ & $9.9 \mathrm{~B}$ & $71.97 \mathrm{~B}$ & $10.21 \mathrm{BC}$ & $70.87 \mathrm{C}$ & $1.011 \mathrm{~A}$ & $1.051 \mathrm{~A}$ & $31.22 \mathrm{CD}$ & $21.43 \mathrm{C}$ \\
\hline & $\mathrm{T} 5$ & $10.1 \mathrm{~A}$ & $72.82 \mathrm{~B}$ & $10.39 \mathrm{~B}$ & $75.98 \mathrm{~B}$ & $1.002 \mathrm{~B}$ & $1.062 \mathrm{~A}$ & $37.01 \mathrm{C}$ & $23.34 \mathrm{~B}$ \\
\hline & $\mathrm{T} 6$ & $9.8 \mathrm{~B}$ & $78.55 \mathrm{~A}$ & $10.51 \mathrm{~B}$ & $83.63 \mathrm{~A}$ & $1.012 \mathrm{~A}$ & $1.034 \mathrm{~B}$ & $28.34 \mathrm{D}$ & $18.17 \mathrm{C}$ \\
\hline & $\mathrm{T} 7$ & $9.7 \mathrm{~B}$ & $70.55 \mathrm{~B}$ & $10.06 \mathrm{C}$ & $78.83 \mathrm{~B}$ & $1.061 \mathrm{~A}$ & $1.067 \mathrm{~A}$ & $31.21 \mathrm{CD}$ & $21.13 \mathrm{~B}$ \\
\hline & $\mathrm{T} 8$ & $10 \mathrm{AB}$ & $73.31 \mathrm{~A}$ & $10.25 \mathrm{~B}$ & $84.48 \mathrm{~A}$ & $1.037 \mathrm{~A}$ & $1.064 \mathrm{~A}$ & $32.45 \mathrm{C}$ & $19.15 \mathrm{C}$ \\
\hline \multirow{9}{*}{ NSG-311 } & T0 & $10.8 \mathrm{~B}$ & $77.25 \mathrm{~A}$ & $9.06 \mathrm{D}$ & $83.76 \mathrm{~B}$ & $0.996 \mathrm{~B}$ & $1.102 \mathrm{~B}$ & $47.34 \mathrm{~A}$ & $29.81 \mathrm{~A}$ \\
\hline & $\mathrm{T} 1$ & $11.6 \mathrm{~A}$ & $73.97 \mathrm{~B}$ & $10.16 \mathrm{BC}$ & $86.81 \mathrm{~A}$ & $0.967 \mathrm{BC}$ & $1.092 \mathrm{C}$ & $32.45 \mathrm{~B}$ & $21.34 \mathrm{~B}$ \\
\hline & $\mathrm{T} 2$ & $10.4 \mathrm{BC}$ & 74.77 B & $10.30 \mathrm{~B}$ & $91.15 \mathrm{~A}$ & $1.011 \mathrm{~A}$ & $1.107 \mathrm{~B}$ & $24.67 \mathrm{C}$ & $17.34 \mathrm{C}$ \\
\hline & $\mathrm{T} 3$ & $11.1 \mathrm{~A}$ & $75.55 \mathrm{~A}$ & $11.40 \mathrm{~A}$ & $92.29 \mathrm{~A}$ & $1.015 \mathrm{~A}$ & $1.121 \mathrm{~A}$ & $21.56 \mathrm{C}$ & $15.23 \mathrm{C}$ \\
\hline & $\mathrm{T} 4$ & $10.7 \mathrm{~A}$ & $74.21 \mathrm{~B}$ & $10.24 \mathrm{~B}$ & $80.28 \mathrm{~B}$ & $0.895 \mathrm{D}$ & $1.102 \mathrm{~B}$ & $23.62 \mathrm{C}$ & $18.19 \mathrm{C}$ \\
\hline & $\mathrm{T} 5$ & $11.0 \mathrm{~A}$ & $77.12 \mathrm{~A}$ & $10.36 \mathrm{~B}$ & $82.56 \mathrm{~B}$ & $1.010 \mathrm{~A}$ & $1.107 \mathrm{~B}$ & $20.71 \mathrm{D}$ & $17.16 \mathrm{C}$ \\
\hline & T6 & $10.9 \mathrm{~B}$ & $80.02 \mathrm{~A}$ & $10.40 \mathrm{~B}$ & $89.69 \mathrm{~A}$ & $1.027 \mathrm{~A}$ & $1.134 \mathrm{~A}$ & $19.34 \mathrm{E}$ & $12.31 \mathrm{D}$ \\
\hline & $\mathrm{T} 7$ & $11.2 \mathrm{~A}$ & $72.82 \mathrm{~B}$ & $10.16 \mathrm{BC}$ & 80.05 B & $0.937 \mathrm{C}$ & $1.115 \mathrm{AB}$ & $21.71 \mathrm{C}$ & $16.15 \mathrm{C}$ \\
\hline & $\mathrm{T} 8$ & $11.1 \mathrm{~A}$ & $74.11 \mathrm{~B}$ & 10.32 B & $82.17 \mathrm{~B}$ & $1.012 \mathrm{~A}$ & $1.117 \mathrm{AB}$ & $25.76 \mathrm{C}$ & $18.91 \mathrm{C}$ \\
\hline \multirow{9}{*}{ HSF-240 } & T0 & $10.2 \mathrm{BC}$ & $79.02 \mathrm{~A}$ & $9.44 \mathrm{E}$ & $91.96 \mathrm{C}$ & $0.855 \mathrm{D}$ & $1.112 \mathrm{C}$ & $36.56 \mathrm{~A}$ & $24.53 \mathrm{~A}$ \\
\hline & $\mathrm{T} 1$ & $11.4 \mathrm{~A}$ & $80.72 \mathrm{~A}$ & $10.98 \mathrm{~B}$ & $97.73 \mathrm{~B}$ & $0.876 \mathrm{D}$ & $1.131 \mathrm{~B}$ & $21.34 \mathrm{C}$ & $17.15 \mathrm{~B}$ \\
\hline & $\mathrm{T} 2$ & $10.1 \mathrm{C}$ & $80.95 \mathrm{~A}$ & $10.70 \mathrm{C}$ & $103.88 \mathrm{~A}$ & $0.875 \mathrm{D}$ & $1.137 \mathrm{~B}$ & $19.19 \mathrm{C}$ & 14.23 BC \\
\hline & $\mathrm{T} 3$ & $11.0 \mathrm{~A}$ & $82.95 \mathrm{~A}$ & $11.67 \mathrm{~A}$ & $107.36 \mathrm{~A}$ & $0.935 \mathrm{~B}$ & $1.132 \mathrm{~B}$ & $20.93 \mathrm{C}$ & $18.31 \mathrm{~B}$ \\
\hline & $\mathrm{T} 4$ & $10.8 \mathrm{~B}$ & $81.05 \mathrm{~A}$ & $10.34 \mathrm{D}$ & $94.84 \mathrm{~B}$ & $0.941 \mathrm{~B}$ & $1.151 \mathrm{~A}$ & $26.54 \mathrm{~B}$ & $19.18 \mathrm{~B}$ \\
\hline & T5 & $11.6 \mathrm{~A}$ & $81.75 \mathrm{~A}$ & $10.48 \mathrm{D}$ & $103.45 \mathrm{~A}$ & $0.952 \mathrm{~B}$ & $1.135 \mathrm{~B}$ & $24.51 \mathrm{~B}$ & $14.25 \mathrm{BC}$ \\
\hline & T6 & $10.6 \mathrm{~B}$ & $83.81 \mathrm{~A}$ & $10.61 \mathrm{~B}$ & $108.50 \mathrm{~A}$ & $0.901 \mathrm{C}$ & $1.152 \mathrm{~A}$ & $15.34 \mathrm{D}$ & $11.12 \mathrm{C}$ \\
\hline & $\mathrm{T} 7$ & $11.2 \mathrm{~A}$ & $75.32 \mathrm{~B}$ & $10.35 \mathrm{D}$ & $101.23 \mathrm{~B}$ & $1.001 \mathrm{~A}$ & $1.135 \mathrm{~B}$ & $18.76 \mathrm{C}$ & $15.67 \mathrm{~B}$ \\
\hline & $\mathrm{T} 8$ & $10.7 \mathrm{~B}$ & $75.52 \mathrm{~B}$ & $11.10 \mathrm{~B}$ & $108.77 \mathrm{~A}$ & $0.977 \mathrm{~A}$ & $1.112 \mathrm{C}$ & $23.44 \mathrm{~B}$ & $12.45 \mathrm{C}$ \\
\hline \multirow{9}{*}{ NSG-555 } & T0 & $10.9 \mathrm{C}$ & $77.50 \mathrm{~B}$ & $10.01 \mathrm{D}$ & $100.26 \mathrm{C}$ & $0.796 \mathrm{E}$ & $1.123 \mathrm{~B}$ & $37.45 \mathrm{~A}$ & $34.56 \mathrm{~A}$ \\
\hline & $\mathrm{T} 1$ & $12.6 \mathrm{~A}$ & $81.25 \mathrm{~A}$ & $11.45 \mathrm{~B}$ & 106.97 B & $0.806 \mathrm{DE}$ & $1.141 \mathrm{AB}$ & $25.67 \mathrm{~B}$ & $20.91 \mathrm{~B}$ \\
\hline & $\mathrm{T} 2$ & $11.7 \mathrm{~B}$ & $81.91 \mathrm{~A}$ & $11.13 \mathrm{BC}$ & $110.18 \mathrm{AB}$ & $0.812 \mathrm{D}$ & $1.132 \mathrm{~B}$ & $24.21 \mathrm{~B}$ & $23.56 \mathrm{~B}$ \\
\hline & $\mathrm{T} 3$ & $12.0 \mathrm{AB}$ & $83.52 \mathrm{~A}$ & $12.12 \mathrm{~A}$ & $116.85 \mathrm{~A}$ & $0.822 \mathrm{D}$ & $1.161 \mathrm{~A}$ & $26.78 \mathrm{~B}$ & $18.23 \mathrm{BC}$ \\
\hline & $\mathrm{T} 4$ & $11.4 \mathrm{~B}$ & $82.72 \mathrm{~A}$ & $11.66 \mathrm{~B}$ & 104.74 B & $0.841 \mathrm{C}$ & $1.132 \mathrm{~B}$ & $22.34 \mathrm{BC}$ & $16.34 \mathrm{C}$ \\
\hline & T5 & $11.4 \mathrm{~B}$ & $83.67 \mathrm{~A}$ & $10.76 \mathrm{C}$ & $111.02 \mathrm{~A}$ & $0.852 \mathrm{~B}$ & $1.157 \mathrm{~A}$ & $23.46 \mathrm{~B}$ & $17.18 \mathrm{C}$ \\
\hline & $\mathrm{T} 6$ & $12.0 \mathrm{AB}$ & $83.05 \mathrm{~A}$ & $11.44 \mathrm{~B}$ & $113.95 \mathrm{~A}$ & $0.912 \mathrm{~A}$ & $1.152 \mathrm{~A}$ & $19.45 \mathrm{C}$ & 19.18 BC \\
\hline & $\mathrm{T} 7$ & $11.7 \mathrm{~B}$ & $82.42 \mathrm{~A}$ & $10.69 \mathrm{C}$ & 106.68 B & 0.847 B & $1.132 \mathrm{~B}$ & $23.68 \mathrm{~B}$ & $21.34 \mathrm{~B}$ \\
\hline & $\mathrm{T} 8$ & $11.0 \mathrm{C}$ & $83.82 \mathrm{~A}$ & $11.21 \mathrm{~B}$ & $113.08 \mathrm{~A}$ & $0.881 \mathrm{AB}$ & $1.123 \mathrm{~B}$ & $21.39 \mathrm{BC}$ & $22.17 \mathrm{~B}$ \\
\hline
\end{tabular}

Capital alphabets show the significant variations among treatment means

\section{Antioxidant activities}

Antioxidant activities i.e. peroxidase (POD) and catalases (CAT) were determined by evaluating the stimulatory effects of different chemical ripeners. Effect of chemical ripeners was highly significant on POD activities in sugarcane (Table 1). Higher POD activities were noted in cultivar HSF-242 and lower was present in HSF-240 (Fig. 1E). Higher POD value (1.06) was noted by the applications of Glyphosate $\left(0.05 \mathrm{~L} \mathrm{ha}^{-1}\right)$ + Ethephon $\left(0.05 \mathrm{~L} \mathrm{ha}^{-1}\right)$. Treatments in combinations had high POD values as compared to separate treatments. For CAT activities also a highly significant effect of chemical ripeners was observed (Table 2). Higher value of CAT (1.16) was noted in 
NSG-555 with the applications of Ethephon $\left(0.05 \mathrm{~L} \mathrm{ha}^{-1}\right)$ and HSF-242 cultivar showed lower CAT activities (Fig. $1 F$ ).

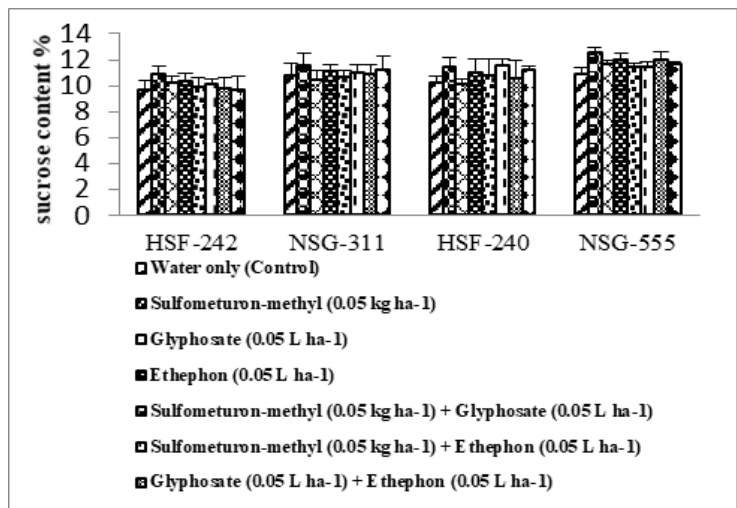

A: Sucrose contents (\%)

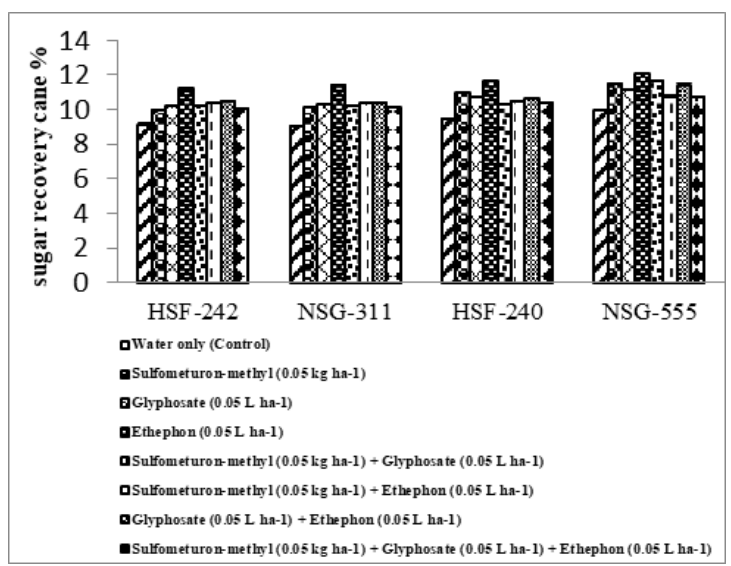

C: Sugar recovery \% cane

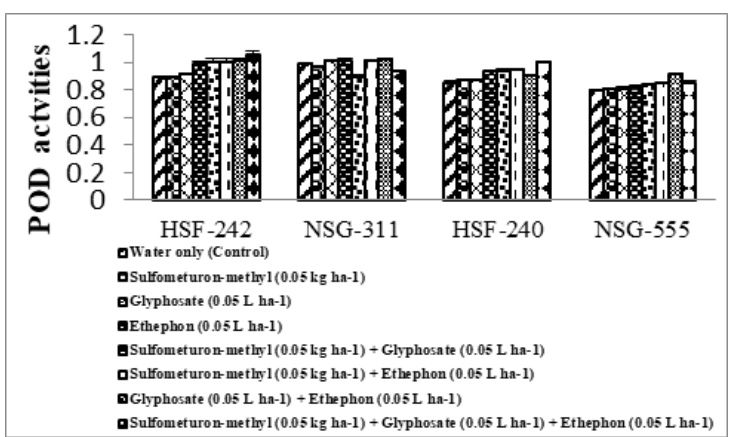

E: POD $(\mu \mathrm{g} / \mathrm{g}$ of FW)

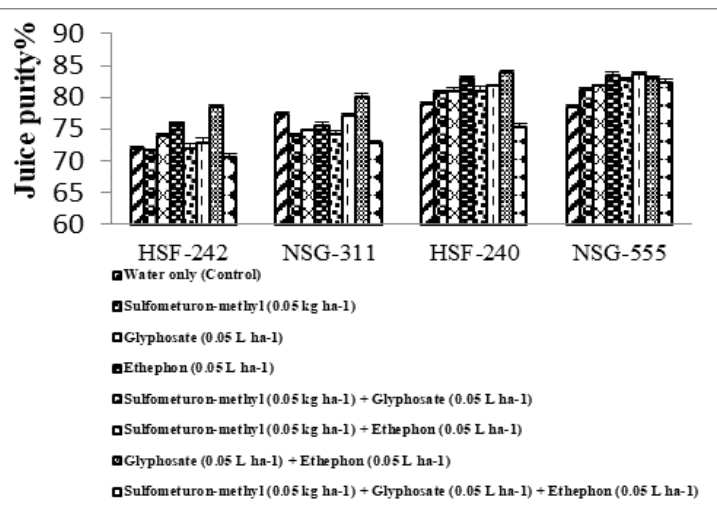

B: Juice purity (\%)

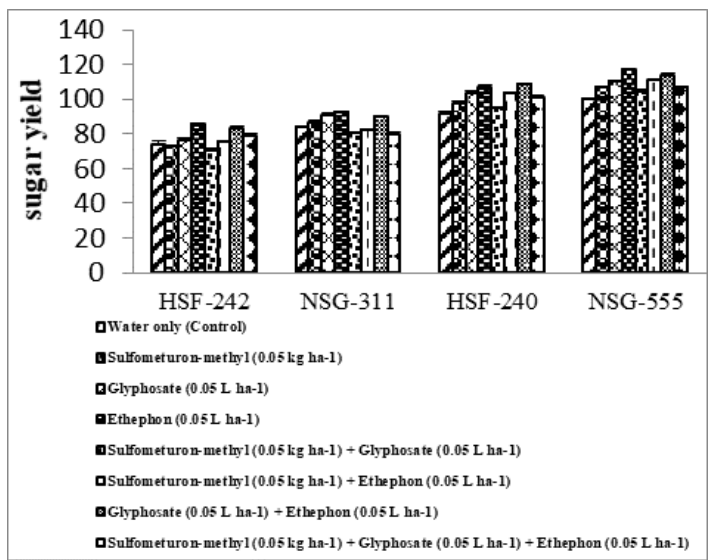

D: Sugar yield (t ha' $\left.{ }^{-1}\right)$

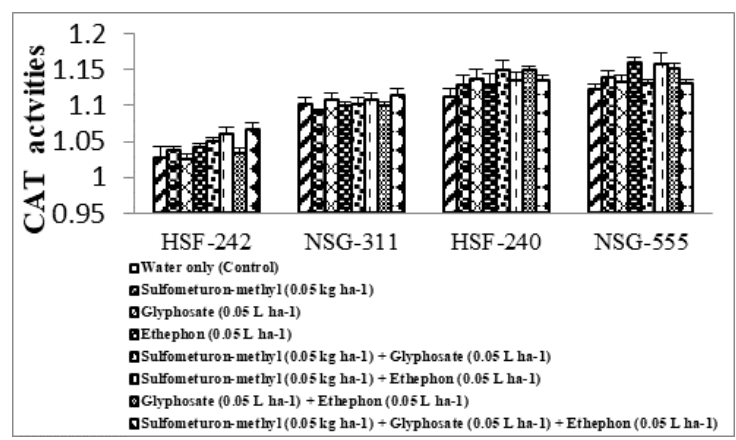

F: CAT ( $\mu \mathrm{mol} / \mathrm{g}$ of FW)

Figure 1. Effect of chemical ripeners on quality attributes and antioxidant activities of sugarcane

\section{Potassium $\left(K^{+}\right)$contents}

Means squares from ANOVA showed highly significant effects on $\mathrm{K}^{+}$accumulation in the juice of sugarcane (Table 1). There were high contents of $\mathrm{K}^{+}$in controls for the cultivars that reduced significantly with the applications of chemical cultivars (Fig. 2A). Sulfometuron-methyl $\left(0.05 \mathrm{~kg} \mathrm{ha}^{-1}\right)+$ Ethephon $\left(0.05 \mathrm{~L} \mathrm{ha}^{-1}\right)$ treatments showed 
maximum reduction for $\mathrm{K}^{+}$(Table 2). Higher $\mathrm{K}^{+}$contents $(15.34 \mathrm{mM})$ was noted in HSF-240 with the applications of Sulfometuron-methyl $\left(0.05 \mathrm{~kg} \mathrm{ha}^{-1}\right)+$ Ethephon $\left(0.05 \mathrm{~L} \mathrm{ha}^{-1}\right)$.

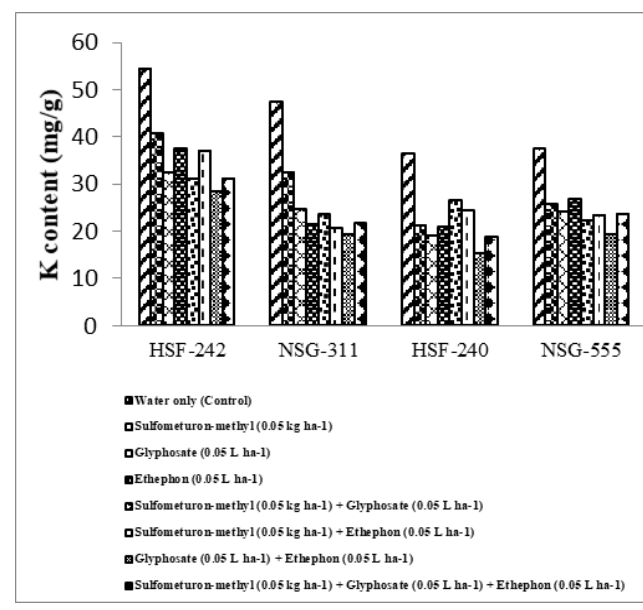

$\mathrm{A}: \mathrm{K}^{+}$contents in juice

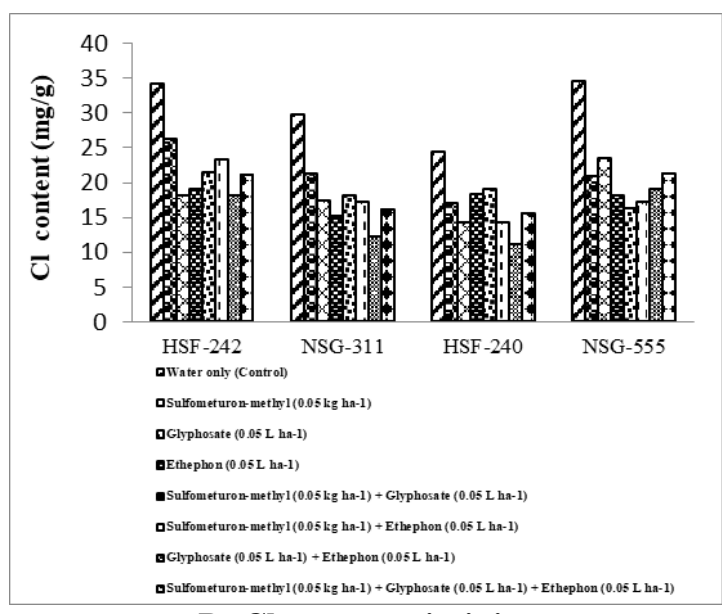

B: $\mathrm{Cl}^{-}$contents in juice

Figure 2. Effect of chemical ripeners on ionic concentrations of sugarcane

\section{Chloride $\left(\mathrm{Cl}^{-}\right)$contents}

Chemical ripeners had highly significant effects on $\mathrm{Cl}^{-}$accumulation in cane juice (Table 1). Pattern of $\mathrm{Cl}^{-}$accumulation was similar as in the case of $\mathrm{K}^{+}$contents in juice of sugarcane with the applications of chemical ripeners (Table 2). Sulfometuron-methyl $\left(0.05 \mathrm{~kg} \mathrm{ha}^{-1}\right)+$ Ethephon $\left(0.05{\left.\mathrm{~L} . h a^{-1}\right)}^{-1}\right.$ treatments showed maximum reduction in $\mathrm{Cl}^{-}$in all the cultivars. Lower $\mathrm{Cl}^{-}$contents $(11.12 \mathrm{mM})$ were noted in HSF-240 with the applications of Sulfometuron-methyl $\left(0.05 \mathrm{~kg} \mathrm{ha}^{-1}\right)+$ Ethephon $\left(0.05 \mathrm{~L} \mathrm{ha}^{-1}\right)$. There were higher contents of $\mathrm{Cl}^{-}$in controls that reduced significantly with the applications of chemical cultivars (Fig. 2B).

\section{Ethylene production in leaf sheath}

Data related to ethylene production in leaf sheath is given in Table 3. Data of ethylene production was collected at the time of treatment and then at maturity. Ethylene production reduced significantly at maturity. Maximum reduction in ethylene production was noted in NSG-555. At maturity, there were much variations in ethylene production as compared to the data collected at the time of treatment. Low ethylene production resulted in the maturity of cane crop.

\section{Pearson correlation}

Correlation was calculated for ethylene, $\mathrm{K}^{+}$and $\mathrm{Cl}^{-}$contents, sucrose contents and sugar yielding attributes under the influence of chemical ripeners. The correlations are shown in Table 4. There was a negative correlation of Ethylene with $\mathrm{K}^{+}$and $\mathrm{Cl}^{-}$and positive with sucrose, sugar recovery and sugar yield. Which showed that ethylene has direct relation with sugar yielding attributes, when the concentration of ethylene increased then sugarcane had maximum sugar production indicating its maturity. $\mathrm{K}^{+}$ contents positive relation with $\mathrm{Cl}^{-}$showed cation and anion direct relationship. 


$$
-70 \text { - }
$$

Table 3. Determination of ethylene production in leaf sheaths (mean values from 2 nd to 5 th leaf) of sugarcane under the applications of chemical ripeners

\begin{tabular}{|c|c|c|c|}
\hline \multirow{2}{*}{ Cultivar } & \multirow{2}{*}{ Treatments } & \multicolumn{2}{|c|}{ Production of ethylene $\left(\mathrm{nL} \cdot \mathrm{g}^{-1} \cdot \mathrm{h}^{-1} \times \mathbf{1 0}^{-4}\right)$} \\
\hline & & At treatment time & At harvest stage \\
\hline \multirow{9}{*}{ HSF-242 } & T0 & $21 \pm 1.12 \mathrm{AB}$ & $104 \pm 1.51 \mathrm{D}$ \\
\hline & $\mathrm{T} 1$ & $28 \pm 1.23 \mathrm{~A}$ & $233 \pm 1.04 \mathrm{~B}$ \\
\hline & $\mathrm{T} 2$ & $22 \pm 1.67 \mathrm{AB}$ & $227+2.10 \mathrm{~B}$ \\
\hline & $\mathrm{T} 3$ & $27 \pm 1.75 \mathrm{~A}$ & $231 \pm 1.12 \mathrm{~B}$ \\
\hline & $\mathrm{T} 4$ & $29 \pm 2.10 \mathrm{~A}$ & $216 \pm 2.11 \mathrm{C}$ \\
\hline & T5 & $29 \pm 1.11 \mathrm{~A}$ & $254 \pm 2.34 \mathrm{~A}$ \\
\hline & T6 & $21 \pm 1.76 \mathrm{AB}$ & $223 \pm 1.98 \mathrm{~B}$ \\
\hline & $\mathrm{T} 7$ & $23 \pm 1.23 \mathrm{~B}$ & $209 \pm 1.08 \mathrm{C}$ \\
\hline & $\mathrm{T} 8$ & $25 \pm 1.83 \mathrm{~B}$ & $261 \pm 1.66 \mathrm{~A}$ \\
\hline \multirow{9}{*}{ NSG-311 } & T0 & $23 \pm 1.01 \mathrm{~A}$ & $98 \pm 1.19 \mathrm{E}$ \\
\hline & $\mathrm{T} 1$ & $22 \pm 1.43 \mathrm{~B}$ & $207 \pm 2.01 \mathrm{C}$ \\
\hline & $\mathrm{T} 2$ & $20 \pm 1.22 \mathrm{~B}$ & $231 \pm 1.08 \mathrm{~B}$ \\
\hline & $\mathrm{T} 3$ & $26 \pm 1.83 \mathrm{~A}$ & $186 \pm 2.11 \mathrm{D}$ \\
\hline & $\mathrm{T} 4$ & $26 \pm 1.56 \mathrm{~A}$ & $257 \pm 1.23 \mathrm{~A}$ \\
\hline & $\mathrm{T} 5$ & $23 \pm 1.09 \mathrm{~A}$ & $204 \pm 1.56 \mathrm{C}$ \\
\hline & T6 & $21 \pm 1.12 \mathrm{~B}$ & $214 \pm 1.23 \mathrm{BC}$ \\
\hline & $\mathrm{T} 7$ & $24 \pm 1.56 \mathrm{~A}$ & $176 \pm 1.01 \mathrm{D}$ \\
\hline & $\mathrm{T} 8$ & $23 \pm 1.34 \mathrm{~A}$ & $210 \pm 1.66 \mathrm{BC}$ \\
\hline \multirow{9}{*}{ HSF-240 } & T0 & $29 \pm 1.45$ & $78 \pm 1.04 \mathrm{D}$ \\
\hline & $\mathrm{T} 1$ & $27 \pm 2.31 \mathrm{C}$ & $186 \pm 1.11 \mathrm{BC}$ \\
\hline & $\mathrm{T} 2$ & $33 \pm 1.23 \mathrm{~B}$ & $190 \pm 1.45 \mathrm{BC}$ \\
\hline & $\mathrm{T} 3$ & $23 \pm 1.54 \mathrm{CD}$ & $166 \pm 1.23 \mathrm{C}$ \\
\hline & $\mathrm{T} 4$ & $26 \pm 2.45 \mathrm{C}$ & $201 \pm 2.01 \mathrm{~A}$ \\
\hline & $\mathrm{T} 5$ & $32 \pm 1.86 \mathrm{~B}$ & $194 \pm 1.54$ B \\
\hline & T6 & $38 \pm 1.23 \mathrm{~A}$ & $204 \pm 1.23 \mathrm{~A}$ \\
\hline & $\mathrm{T} 7$ & $37 \pm 1.64 \mathrm{~A}$ & $198 \pm 1.45 \mathrm{AB}$ \\
\hline & $\mathrm{T} 8$ & $31 \pm 2.03 \mathrm{~B}$ & $183 \pm 1.19 \mathrm{BC}$ \\
\hline \multirow{9}{*}{ NSG-555 } & T0 & $18 \pm 1.01 \mathrm{C}$ & $88 \pm 1.12 \mathrm{E}$ \\
\hline & $\mathrm{T} 1$ & $16 \pm 1.43 \mathrm{C}$ & $203 \pm 1.04 \mathrm{~B}$ \\
\hline & $\mathrm{T} 2$ & $20 \pm 1.24 \mathrm{~B}$ & $187 \pm 2.11 \mathrm{~B}$ \\
\hline & $\mathrm{T} 3$ & $14 \pm 1.45 \mathrm{D}$ & $154 \pm 1.76 \mathrm{D}$ \\
\hline & $\mathrm{T} 4$ & $17 \pm 1.67 \mathrm{C}$ & $209 \pm 1.09 \mathrm{~A}$ \\
\hline & T5 & $25 \pm 1.11 \mathrm{~A}$ & $194 \pm 1.11 \mathrm{BC}$ \\
\hline & T6 & $22 \pm 1.03 \mathrm{~A}$ & $201 \pm 1.23 \mathrm{~B}$ \\
\hline & $\mathrm{T} 7$ & $21 \pm 1.34 \mathrm{~B}$ & $219 \pm 1.43 \mathrm{~A}$ \\
\hline & $\mathrm{T} 8$ & $19 \pm 1.14 \mathrm{~B}$ & $183 \pm 2.11 \mathrm{~B}$ \\
\hline
\end{tabular}

Different alphabets show the significant variations

\section{Discussion}

Results indicated that chemical ripeners had significant effect for the enhancement of sugar yield and early ripening of sugarcane. It might be due to the conversion of cane formation and elongation phase into ripening phase by Ethephon, Glyphosate and Sulfometuron-methyl. Similarly, Karmollachaab et al. (2016) noted a beneficial 
increase in juice pole and brix value and comparatively high sugar yield can be achieved by applying Glyphosate and Ethephon. Different responses of sugarcane varieties were noted for different chemical ripeners like the effect of ethephon as a ripener was studied by many researchers (Silva et al., 2007). The reducing ability of ethephon on flowering reduces the cane stalk and increases the productivity of sugar. Ethophon anticipated harvesting stage with 21 days of applications and increased sugar yield (Caputo et al., 2008; Dalley and Richard, 2010).

Table 4. Pearson correlation coefficients ( $r$ ) of sugarcane variables under the applications of chemical ripeners

\begin{tabular}{c|c|c|c|c|c}
\hline & Ethylene & K Contents & Cl Contents & Sucrose contents & Sugar recovery \% cane \\
\hline K Contents & -0.891526812 & & & & \\
Cl Contents & -0.777345533 & 0.922058378 & & & \\
Sucrose Contents & 0.305346091 & -0.078612401 & -0.031781141 & & \\
Sugar recovery \% cane & 0.668635845 & -0.571810994 & -0.548581377 & 0.0267 & \\
Sugar yield & 0.857606233 & -0.790316064 & -0.79438101 & 0.22931 & 0.672011 \\
\hline
\end{tabular}

$\mathrm{r}$ value $>0$ indicates positive correlation

Results demonstrated consistent improvement in sugarcane brix, pol with the applications of chemical ripeners. Sulfometuron-methyl can also result a reduction in pith process (50 to $60 \%$ ) for cane maturity. An increase in pol $(1.26 \%$ ) and earlier ripening of 21 days and reduction in reducing sugar was observed by the application of sulfometuron-methyl (Caputo et al., 2008). Su et al. (1992) indicated that glyphosate brought about an expansion in sucrose content in the stalk only five days after application.

Different factors such as variety of sugarcane, time of application of ripeners, stage of plant life cycle, application rate of chemical, type or combination of ripener can affect sugarcane ripening by chemical ripeners. In different studies, the utilization of substance ripeners, for example, ethephon and glyphosate had been investigated with positive impacts in repining (Solomon and $\mathrm{Li}, 2004$ ). Li et al. (2004) showed that compound ripener in sugarcane had an effect for high sugar accumulation in sugarcane. Watanabe et al. (2016) showed that $\mathrm{K}^{+}$and $\mathrm{Cl}^{-}$contents were the most abundant cation and anion present in sugarcane and both are negatively correlated with sucrose concentration. High $\mathrm{K}^{+}$and $\mathrm{Cl}^{-}$contents had significant effects on lowering sucrose concentration. Chemical ripeners can affect the ethylene production to enhance the maturity.

Plant hormone ethylene has a significant regulatory role in the ripening of many fruits with important contribution in nutritional and fiber contents. Molecular exploration of the role of ethylene in fruit ripening has led to the affirmation of mechanisms of ethylene perception (Barry and Giovannoni, 2007). Ethylene production changes when a fruit reaches maturity. The negative effects of ethylene on quality of fruit ripening are related to shifting or speeding up the natural processes of growth, maturity and deterioration with age, while the favorable outcomes of ethylene on quality center on generally the same features as the negative effects, but vary in both level and direction (Srividhya and Sujatha, 2017). Cunha et al. (2017) identified potential ethylene target genes and characterized the hormonal changes during ripening, providing insights into the action of ethylene at the site of sucrose accumulation. Changes in ethylene production level, its perception directly or indirectly regulate the lifespan of plants (Iqbal et al., 2017). 


\section{Conclusion}

It was concluded that chemical ripeners enhanced early cane maturity with high sugar production by increasing the ethylene production and reducing the $\mathrm{K}^{+}$and $\mathrm{Cl}^{-}$ contents. Chemical ripeners can be used for early cane maturity for these sugarcane cultivars in Pakistan.

\section{Recommendation}

It is suggested that chemical ripeners can be useful to induce early cane ripening for profitable productivity in sugarcane.

\section{REFERENCES}

[1] Almodares, A., Usofzadeh, M., Daneshvar, M. (2013): Effect of nitrogen and ethephon on growth parameters, carbohydrate contents and bioethanol production from sweet sorghum. - Sugar Tech 15(3): 300-304.

[2] Barry, C. S., Giovannoni, J. J. (2007): Ethylene and fruit ripening. - Journal of Plant Growth Regulation 26: 143-159.

[3] Caputo, M. M., Beauclair, E. G. F., Silva, M. D. A., Piedade, S. M. D. S. (2008): Response of sugarcane genotypes to the application of maturation inducers. - Bragantia 67(1): 15-23.

[4] Chance, M., Maehly, A. C. (1955): Assay of catalases and peroxidases. - Methods in Enzymology 2: 764-817.

[5] Chong, B. F., Mills, E., Bonnett, G. D., Gnanasambandam, A. (2010): Early exposure to ethylene modifies shoot development and increases sucrose accumulation rate in sugarcane. - Journal of Plant Growth Regulation 29(2): 149-163.

[6] Cunha, C. P., Roberto, G. G., Vicentini, R., Lembke, C. G., Souza, G. M., Ribeiro, R. V., Machado, E. C., Lagoa, A. M. M. A., Menossi, M. (2017): Ethylene-induced transcriptional and hormonal responses at the onset of sugarcane ripening. - Scientific Reports 7: 43364.

[7] Dalley, C. D., Richard, E. P. (2010): Herbicides as ripeners for sugarcane. - Weed Science 58(3): 329-333.

[8] De Almeida, S. M., Caputo, M. M. (2012): Ripening and the Use of Ripeners for Better Sugarcane Management. - In: Marin, F. (ed.) Crop Management - Cases and Tools for Higher Yield and Sustainability. In Tech Europe, Rijeka. https://doi.org/10.5772/28958.

[9] El-Hamd, A. S., Bekheet, M. A., Gadalla, A. F. I. (2013): Effect of chemical ripeners on juice quality, yield and yield components of some sugarcane varieties under the conditions of Sohag Governorate. - American-Eurasian Journal of Agriculture and Environment Science 13(11): 1458-1464.

[10] Goscinny, S., Hanot, V. (2012): Glyphosate in pouch states. - Scientific Institute of Public Health, Pesticides Unit Labinfo 7: 12-16.

[11] Horwitz, W. (1976): Official Methods of Analysis of the Association of Official Analytical Chemists. 12th Ed. - Association of Official Analytical Chemists, Washington, DC. https://doi.org/10.1002/jps.2600650148.

[12] Iqbal, N., Khan, N. A., Ferrante, A., Trivellini, A., Francini, A., Khan, M. I. R. (2017): Ethylene role in plant growth, development and senescence: interaction with other phytohormones. - Frontier in Plant Science 8: 475.

[13] Karmollachaab, A., Bakhshandeh, A., Telavat, M. M., Moradi, F., Shomeili, M. (2016): Sugarcane yield and technological ripening responses to chemical ripeners. - Sugar Tech 18(3): 285-291. 
[14] Legendre, B. L., Henderson, M. T. (1972): The history and development of sugarcane yield calculations. $\quad-\quad$ Proceeding ASSCT 2: 10-18. https://doi.org/10.1177/1032373211435501.

[15] Lee, T. T., Dumas, T. (1982): Effect of glyphosate on ethylene production in tobacco callus. - Plant Physiology 72: 855-857.

[16] Leite, G. H. P., Crusciol, C. A. C., Silva, M. D. A., Venturini, F. (2009): Ripeness and technological quality of early harvest sugarcane variety RB855453. - Bragantia 68(3): 781-787.

[17] Leite, G. H. P., Crusciol, C. A. C., Siqueira, G. F. D., Silva, M. D. A. (2010): Technological quality in different sections of the stalk and productivity of sugarcane under the effect of ripeners. - Bragantia 69(4): 861-870.

[18] Li, Y. R., Zhu, Q. Z., Ye, Y. P., Wang, W. Z., Yang, L. T., Pan, L. Q., Xu, T. (2004): Sugarcane Ripening Trials with Glyphosate-Borate Complex in Commercial Plantation of Shansi County. - In: Li, Y. R., Solomon, S. (eds.) Proceedings of the International Symposium on Sustainable Sugarcane and Sugar Production Technology. Nanning, China. China Agriculture Press, Beijing. https://doi.org/10.3133/b540o.

[19] Ma, J. K., Chikwamba, R., Sparrow, P., Fischer, R., Mahoney, R., Twyman, R. M. (2005): Plant-derived pharmaceuticals - the road forward. - Trends in Plant Sciences 10(12): 580-585.

[20] Manzoor, R., Maken, A., Culas, R. (2019): Sustaining agricultural production in Pakistan: obstacles and prospects. - Current Political and Economics of Middle East 10(3): 331356.

[21] McDonald, M., Jackson, P. (2001): The effect of ripeners on the CCS of 47 sugarcane varieties in the burdekin. - Proceedings of the Australian Society of Sugar Cane Technology 23: 102-108.

[22] Neliana, I. R., Sawitri, W. D., Ermawati, N., Handoyo, T., Sugiharto, B. (2019): Development of Allergenicity and Toxicity Assessment Methods for Evaluating Transgenic Sugarcane Overexpressing Sucrose-Phosphate Synthase. - Agronomy 9(1): 23.

[23] Silva, M. D. A., Gava, G. J. D. C., Caputo, M. M., Pincelli, R. P., Jerônimo, E. M., Cruz, J. C. S. (2007): These cruise control regulators have the potential to be developed and manufactured separately. - Bragantia 66(4): 545-552.

[24] Solomon, S., Li, Y. R. (2004): Chemical ripening of sugarcane: global progress and recent developments in China. - Sugar Tech 6(4): 241-249.

[25] Srividhya, V., Sujatha, K. (2017): Role of ethylene in fruits ripening process. - 2017 International Conference on Energy, Communication, Data Analytics and Soft Computing (ICECDS). Chennai, India. https://doi.org/10.1007/978-94-009-6178-4_45.

[26] Su, L. Y., Cruz, A. D., Moore, P. H., Maretzki, A. (1992): The relationship of glyphosate treatment to sugar metabolism in sugarcane: new physiological insights. - Journal of Plant Physiology 140(2): 168-173.

[27] Van Heerden, P. D., Mbatha, T. P., Ngxaliwe, S. (2015): Chemical ripening of sugarcane with trinexapac-ethyl (Moddus ${ }^{\circledR}$ ) Mode of action and comparative efficacy. - Field Crops Research 181: 69-75.

[28] Watanabe, K., Nakabarua, M., Tairaa, E., Uenoa, M., Kawamitsua, Y. (2016): Relationships between nutrients and sucrose concentrations in sugarcane juice and use of juice analysis for nutrient diagnosis in Japan. - Plant Production Science 19(2): 215-222. 